\title{
PROJETO EXTENSIONISTA SOBRE CÂNCER DE MAMA E OSTEOPOROSE PARA IDOSOS: relato de experiência
}

\author{
Kethylen da Silva de Jesus \\ Instituto Federal de Santa Catarina campus Florianópolis \\ kethylen.s@aluno.ifsc.edu.br \\ Fernando Rockembach Mazuim \\ Instituto Federal de Santa Catarina campus Florianópolis \\ fernando.rm06@aluno.ifsc.edu.br
}

Leticia Nunes Faustino

Instituto Federal de Santa Catarina campus Florianópolis leticia.nf@aluno.ifsc.edu.br

Pamella Aires Feldhaus

Instituto Federal de Santa Catarina campus Florianópolis pamella.f@aluno.ifsc.edu.br

Caroline de Medeiros

Instituto Federal de Santa Catarina campus Florianópolis carol@ifsc.edu.br

Charlene da Silva

Instituto Federal de Santa Catarina campus Florianópolis charlene.silva@ifsc.edu.br

\begin{abstract}
Resumo
O presente artigo relata como foi implementado o projeto Radiologia na Comunidade, que propõe- se a conscientização e aprimoração do conhecimento de uma comunidade de Florianópolis-SC, sobre os principais aspectos relacionados ao câncer de mama e a osteoporose. O objetivo deste estudo é relatar a experiência dos discentes do curso de graduação em Radiologia do Instituto Federal de Educação, Ciência e Tecnologia de Santa Catarina - Campus Florianópolis (IFSC/SC) em uma ação extensionista no Conselho Comunitário Fazenda do Rio Tavares, para o Grupo de Convivência Para Idosos empregando as definições das patologias, bem como da anatomia básica das estruturas envolvidas, formas de prevenção, fatores de risco, sinais e sintomas, métodos diagnósticos e tratamentos de cada patologia. Materiais e métodos: Para alcançar o objetivo foi desenvolvido um questionário básico onde era possível informar se já teve/tem alguma das patologias e/ou se alguém da família já teve/tem umas das patologias, durante a palestra expositiva e dialogada, com duração de 60 minutos foram utilizados recursos audiovisuais como slides e vídeos para o desenvolvimento da apresentação, foram disponibilizados folders informativos e realizadas dinâmicas como sorteio e bingo. Com base nos dados obtidos através do questionário aplicado com os 28 participantes que compareceram no dia da ação extensionista, observou-se que desse total, 8 apresentavam como grau de escolaridade o ensino fundamental incompleto, sendo esse o grau de ensino com o maior número de representantes, 1 era analfabeto e 5 possuíam o ensino superior completo. Em relação ao gênero, 25 eram do sexo feminino. Nas questões que envolveram as patologias apresentadas, 6 pessoas já tiveram algum familiar com câncer de mama e 14 com familiares
\end{abstract}


portadores de osteoporose e, informado apenas por mulheres, 3 manifestaram ter desenvolvido o câncer de mama e 13 apresentaram osteoporose. Com isso, conclui-se que transmitir importantes informações sobre essas patologias às comunidades, visando que ambas acometem uma grande parcela da população, tem potencial de contribuir com a qualidade de vida dos participantes bem como promover o enriquecimento acadêmico do estudante e proporcionar um momento de troca de conhecimento e experiências entre todos presentes na palestra.

Palavras-chave: Extensão universitária. Neoplasias da mama. Osteoporose, Idosos.

\title{
EXTENSIONIST DESIGN ON BREAST CANCER AND OSTEOPOROSIS FOR ELDERLY: experience report
}

\begin{abstract}
This article reports on the implementation of the Radiology project in the community, which proposes the awareness and improvement of the knowledge of a community of Florianópolis-SC, on the main aspects related to breast cancer and osteoporosis. The objective of this study is to report the experience of students of the undergraduate course in Radiology of the Instituto Federal de Educação, Ciência e Tecnologia de Santa Catarina Campus Florianópolis (IFSC) in an extensionist action in the Community Council Fazenda do Rio Tavares, for the Elderly Living Group using the definitions of the pathologies, as well as the basic anatomy of the involved structures, prevention forms, risk factors, signs and symptoms, diagnostic methods and treatments of each pathology. Materials and methods: In order to reach the objective, a basic questionnaire was developed in which it was possible to inform if the patient had any of the pathologies and / or if one of the family had any of the pathologies during the 60 minutes were used audiovisual resources such as slides and videos for the development of the presentation, informative folders were made available and dynamics such as raffle and bingo. Based on the data obtained through the questionnaire applied with the 28 participants who attended on the day of the extension action, it was observed that of this total, 8 had as incomplete elementary school education, being that the degree of education with the highest number of 1 were illiterate and 5 had completed tertiary education. Regarding gender, 25 were female. In the questions that involved the pathologies presented, 6 had already had a family member with breast cancer and 14 with relatives with osteoporosis and, informed only by women, 3 reported having developed breast cancer and 13 had osteoporosis. With this, it is concluded that transmitting important information about these pathologies to the communities, aiming at both involving a large portion of the population, has the potential to contribute to the quality of life of the participants as well as promote the student's academic enrichment and provide a moment of exchange of knowledge and experiences among all present in the lecture.
\end{abstract}

Keywords: University Extension. Breast neoplasms. Osteoporosis, Elderly.

\section{PROYECTO EXTENSIONISTA SOBRE CÁNCER DE MAMA Y OSTEOPOROSISPARA AÑOS: relato de experiencia}

\begin{abstract}
Resumen
El presente artículo se relaciona como se implementa o proyecta Radiologia en la Comunidad, que se propone una concientización y una estrategia de diseño de la comunidad de Florianópolis-SC, sobre los principios y aspectos relacionados con el cáncer de mama y la osteoporosis. El objetivo de este estudio es relatar la experiencia de los alumnos (as) del curso de graduación en Radiología del Instituto Federal de Educación, Ciencia y Tecnología de Santa Catarina del Cámpus Florianópolis (IFSC / SC) en una acción extensionista en el Consejo Comunitario Hacienda del Río Tavares para el Grupo
\end{abstract}

Cidadania em Ação; Revista de Extensão e Cultura, Florianópolis (SC), v. 3, n. 2, jul./dez. 2019 
de Convivencia para ancianos empleando las definiciones de las patologías, así como de la anatomía básica de las estructuras involucradas, formas de prevención, factores de riesgo, signos y síntomas, métodos diagnósticos y tratamientos de cada patología. Materiales y métodos: Para alcanzar el objetivo fue desarrollado un cuestionario básico donde era posible informar si ya tuvo / tiene alguna de las patologías y / o si alguien de la familia ya tuvo o tiene unas de las patologías, se utilizaron recursos audiovisuales como diapositivas y videos para el uso el desarrollo de la presentación, se ofrecieron carpetas con informaciones y realizadas dinámicas (sorteo y bingo). Con base en los datos obtenidos a través del cuestionario aplicado con los 28 participantes que asistieron el día de la acción extensionista, se observó que de ese total, 8 presentaban como grado de escolaridad la enseñanza fundamental incompleta, siendo ese el grado de enseñanza con el mayor número de enseñanza los representantes, 1 era analfabeto y 5 poseían la enseñanza superior completa. En cuanto al género, 25 eran del sexo femenino. En las cuestiones que involucraron las patologías presentadas, 6 personas ya tuvieron algún familiar con cáncer de mama y 14 con familiares portadores de osteoporosis e, informado sólo por mujeres, 3 manifestaron haber desarrollado el cáncer de mama y 13 presentaron osteoporosis. Con ello, se concluye que transmitir importantes informaciones sobre esas patologías a las comunidades, visando que ambas acomete una gran parte de la población, tiene potencial de contribuir con la calidad de vida de los participantes así como promover el enriquecimiento académico del estudiante y proporcionar un momento de intercambio de conocimiento y experiencias entre todos presentes en la conferencia.

Palabras clave: Extensión universitaria. Neoplasias de la mama. Osteoporosis, Ancianos. 


\section{INTRODUÇÃO}

De acordo com a Organização Mundial da Saúde (OMS), idoso é todo indivíduo com 60 anos ou mais. O envelhecimento é uma etapa do ciclo vital, marcado por mutações biológicas visíveis e cercado por determinantes sociais que tornam as concepções sobre velhice variáveis de indivíduo para indivíduo, de cultura para cultura, de época para época. Conforme aponta o Instituto Brasileiro de Geografia e Estatística (IBGE), a população idosa com mais de 60 anos em 2019, ultrapassa os 29 milhões e a expectativa do IBGE é que, até 2060, este índice chegue em 73 milhões com 60 anos ou mais, o que representaria um crescimento de $160 \%$.

Os grupos de terceira idade ou grupos de idosos, constituem-se num novo espaço para o extravasamento de emoções, podendo funcionar como canal de comunicação entre idosos e familiares ou entre idosos e profissionais de saúde (MARTINS et al., 2007).

O estudo longitudinal da saúde dos idosos brasileiros (ELSI), rede internacional de pesquisa sobre o envelhecimento, mostra que 75,3\% dos idosos brasileiros dependem do Sistema Único de Saúde (SUS).

Considerando, que com o passar dos anos, os indivíduos estão mais propensos a desenvolver certas doenças, se faz importante o entendimento destas patologias, em vista de promover a prevenção e a detecção precoce. Nesta perspectiva, pode-se citar como doenças que acometem de forma significativa a terceira idade, o câncer de mama e a osteoporose.

O câncer de mama é caracterizado pela multiplicação desordenada das células da mama, da qual as células se tornam anormais e assim formam o tumor, é o tipo de doença mais comum entre as mulheres, principalmente mulheres acima de 50 anos, no Brasil o percentual dessa doença chega a 29\% e no ano de 2016 ocorreram 16.069 mortes de mulheres por câncer de mama no país (INCA, 2019). Visto que esse índice é significativamente alto para uma promoção da prevenção, esse foi um dos tópicos mais abordados no projeto extensionista.

Já, a osteoporose é uma doença crônica progressiva, caracterizada por uma diminuição da massa óssea e rompimento da arquitetura óssea, o que compromete a força do osso e propicia fraturas. De acordo com os dados da Fundação Internacional de Osteoporose (IOF), atinge cerca de dez milhões de pessoas no Brasil e de acordo com o IBGE serão pelo menos 18 milhões em 2020, e de acordo com a médica Cláudia Brazão, a partir dos 50 anos, $30 \%$ das mulheres e $13 \%$ dos homens podem ter algum tipo de fratura por osteoporose ao longo da vida, visto que esse número tende a aumentar, em vista da

Cidadania em Ação; Revista de Extensão e Cultura, Florianópolis (SC), v. 3, n. 2, jul./dez. 2019 
importância dos temas anteriormente discutidos, o ensino superior está englobado nesta temática nos aspectos sociais que tangem a extensão universitária. Esta permite que haja um compartilhamento do conhecimento adquirido da pesquisa desenvolvidos na instituição para com a comunidade, por meio de interação com o mesmo, conhecendo a realidade social do mesmo. A interação com a comunidade é muito importante para a formação profissional e humanizada direcionada para a população brasileira (ALMEIDA; PEREIRA; OLIVEIRA, 2016; UFES, 2019).

Com o objetivo de cumprir seu papel social, discentes e docentes do Curso Superior de Tecnologia CST em Radiologia do Instituto Federal de Educação, Ciência e Tecnologia de Santa Catarina, campus Florianópolis(SC), desenvolveram uma ação extensionista apresentando conteúdos relacionados a câncer de mama e osteoporose. Nota-se a importância de abordar esses temas, principalmente para idosos que possam não ter acesso a informação confiável de como se prevenir e ter os devidos cuidados com respeito destas patologias.

Este projeto extensionista, chamado de Radiologia na Comunidade, ocorre desde de 2006, e, ao longo dos anos, vem sofrendo processo continuado de aperfeiçoamento.

O objetivo deste estudo é relatar a experiência dos discentes do curso de graduação em Radiologia do Instituto Federal de Educação, Ciência e Tecnologia de Santa CatarinaCampus Florianópolis (IFSC/SC) em uma ação extensionista no Conselho Comunitário Fazenda do Rio Tavares, para o Grupo de Convivência Para Idosos empregando as definições das patologias, bem como da anatomia básica das estruturas envolvidas, formas de prevenção, fatores de risco, sinais e sintomas, métodos diagnósticos e tratamentos de cada patologia.

\section{RELATO DE EXPERIÊNCIA}

De acordo com Rodrigues et al. (2013) a extensão universitária traz uma grande contribuição para a sociedade, pois há contato entre comunidade e acadêmicos, assim o que é aprendido em sala se concretiza e dissemina informações úteis para aqueles que os escutam. Dentre esses cursos de tecnologia ofertados pelo IFSC se encontra o Curso Superior de Tecnologia em Radiologia (CST em Radiologia), o qual em seu Projeto Pedagógico de Curso (PPC), tem como matéria obrigatória a Extensão II, que possui como objetivo implementar ações que possibilitem um amplo e efetivo compartilhamento do 
conhecimento aprendido em sala (LOH; GRABOSKY e BASSKUK, 2003).

Com o intuito de levar às comunidades informações sobre duas das doenças que mais atingem a população brasileira, o câncer de mama e a osteoporose, o CST em Radiologia do Instituto Federal de Santa Catarina, campus Florianópolis (SC), possui em sua unidade curricular as matérias de "Densitometria Óssea e Mamografia", em conjunto com essas disciplinas tem uma oferta anual de palestras sobre estes assuntos para a comunidade local escolhida pelos próprios estudante. Nesta unidade curricular, os estudantes reconhecem a importância desses exames diagnósticos para prevenir e detectar precocemente a osteoporose e o câncer de mama. Assim, o projeto integra os universitários e um setor específico da sociedade, as mulheres (MEDEIROS et al., 2014).

O projeto Radiologia na comunidade veio a ser criado por uma das docentes do curso no ano de 2006 e desde então vem sendo aperfeiçoado e trabalhado até os dias de hoje; esse projeto desde então vem ajudado muitas mulheres na região de Florianópolis e Grande Florianópolis-SC. Esse projeto tem como objetivo promover maior contato com do discente (futuro tecnólogo) com o público em geral, oferecendo informação de qualidade sobre o câncer de mama e a osteoporose, abordando sobre os temas como fatores de risco, prevenção e exames para detecção, buscando orientar os que os escutam. Tem como maior público as mulheres, visto que essas doenças acometem muito mais as mulheres que os homens, mas os homens são sempre bem vindos nas palestras. Para escolha da comunidade deve-se levar em conta um grupo com mais de 20 pessoas, com um público preferencialmente idoso e sendo a maioria mulheres.

O projeto de Extensão II tem como objetivo conhecer as necessidades sobre os conhecimentos de doenças como a osteoporose e câncer de mama dos idosos que participam de grupos de terceira idade e assim disseminar conhecimento para os mesmo, os incentivando a se cuidarem, e, ainda, proporcionar aos estudantes uma grande troca de experiências, ocasionando em enriquecimento acadêmico e profissional. Os idosos sofrem com uma série de modificações biológicas, fisiológicas, cognitivas, patológicas e socioeconômicas necessitando assim de uma atenção especial (MARTINS et al., 2007), além de transmitir conhecimento no projeto tem-se uma troca com os idosos juntos com o estudantes, isso é bem destacado por Martins et al. (2007) que destaca a importância de reconhecer que o ciclo da vida (envelhecimento) integra uma série de etapas e vivências entre si e num todo integralizado. 
Para a elaboração deste projeto, o processo de criação se iniciou com a pesquisa sobre os assuntos que seriam abordados na palestras, sendo eles câncer de mama e osteoporose. No primeiro momento tivemos aulas com o objetivos de nos aprofundarmos nos assuntos e nos incentivar a pesquisar os mesmo, buscar artigos para elaboração dos nossos slides e material a ser disponibilizado para a comunidade.

Depois dessas aulas foi elaborado os slides com base nos artigos pesquisados e no caso do câncer de mama baseado inteiramente no site do Instituto Nacional do Câncer (INCA) e a Sociedade Brasileira de Mastologia.

Foi elaborado um folder (Figura 1) com informações sobre a nossa palestra e um banner simples onde foi publicado no Facebook do projeto.

Figura 1 -Palestras oferecidas nas comunidade no ano de 2019:

(a) Folder frente;

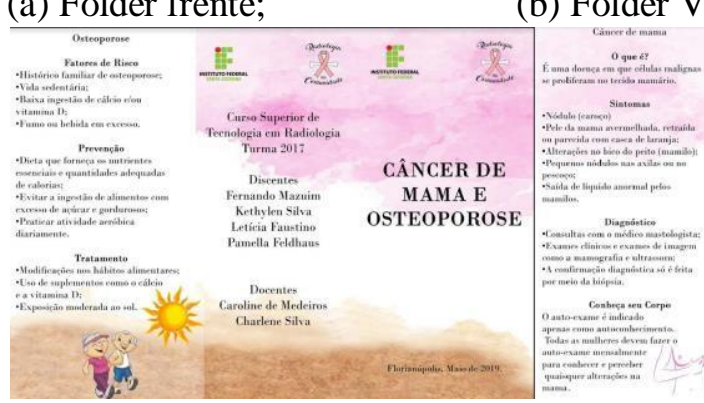

Fonte: Acervo pesquisa (2019). (b) Folder Verso;

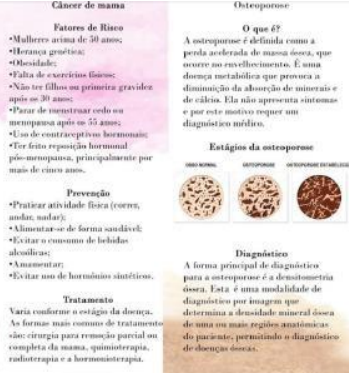

(c) Banner

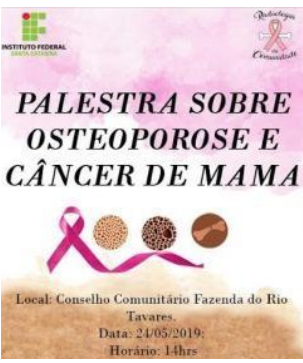

Além de materiais informativos à comunidade em que o projeto foi apresentado, outros discentes que faziam parte da turma também elaboraram matérias sobre os temas para publicação na página do Facebook, podendo assim, alcançar ainda mais pessoas, com informações úteis e confiáveis mesmo que elas não fizessem parte da comunidade escolhida. A página do projeto, Radiologia na Comunidade, do Facebook foi possível dar mais visibilidade ao projeto para que ele possa ter proporções maiores e alcançar vários públicos. Para uma apresentação descontraída, os estudantes realizaram um bingo com prêmios obtidos por meio de doações do comércio local da cidade.

Para avaliar o nível de conhecimento sobre os exames, e a quantidade de pessoas que tem/teve câncer ou teve caso na família, foi elaborado um questionário simples de sim ou não para que os participantes respondessem.

O primeiro contato com a comunidade foi feito no dia 04 de abril de 2019, por meio de comunicação de rede social com a organizadora do projeto Reunião com Grupo de Convivência Para Idosos, que acontecem todas às sextas-feiras no Conselho 
Comunitário da Fazenda do Rio Tavares, Florianópolis-SC, onde a organizadora do grupo aceitou fazer uma reunião no dia 12 de abril de 2019 com os participantes do grupo, para discussão da possibilidade da realização do projeto de extensão. Está reunião foi base para o conhecimento do local/espaço para realização da palestra, foi elaborado também um questionário para que a organizadora do grupo de idosos respondesse, para que os discentes elaborassem e aprimorasse sua palestra de acordo com a comunidade e para entrega do ofício de solicitação para realização do projeto com a comunidade em questão, nesta reunião foi estabelecido que o projeto aconteceria às 14 horas no dia 24 de maio de 2019.

No dia 24 de maio de 2019, os integrantes do projeto e as docentes orientadoras se encaminharam até o conselho comunitário da fazenda do Rio Tavares, localizado no bairro Rio Tavares em Florianópolis-SC, para a apresentação ao Grupo de Convivência Para Idosos. Para apresentação o grupo usa recursos audiovisuais, como: slides e vídeos. O grupo composto por quatro pessoas, foi dividido em duas pessoas por tema, dois para câncer de mama e dois para osteoporose, seguindo esta ordem para apresentação.

\section{CÂNCER DE MAMA}

À apresentação sobre câncer de mama teve início com um conceito básico sobre a anatomia das mamas, mostrando à localização do mamilo, gordura e parte funcional da mama. Em seguida foi explicado sobre o que é câncer de mama, onde foi incentivado a participação dos ouvintes que puderam se expressar sobre o que eles achavam que era o câncer de mama, por meio de um questionário feito aos participantes, estes relatam ser um tumor, após esta interação foi explicado de maneira científica o que é câncer de mama, mostrando por figuras que é uma multiplicação desordenada das células da mama, da qual as células se tornam anormais e assim formam o tumor, e que cada câncer evolui de maneira diferente, uns podem ser mais lentos outros mais rápidos. Usou-se uma referência do INCA onde ele informa que o Câncer de mama é o tipo de doença mais comuns entre as mulheres no mundo e no Brasil, corresponde a cerca de $29 \%$ dos casos novos a cada ano no Brasil (INCA, 2019).

O grupo questionou aos participantes sobre se somente as mulheres têm a possibilidade de ter câncer de mama, os participantes responderam rapidamente que não (uma participante relatou que seu irmão teve câncer de mama), o grupo então informou 
aos participantes que os homens também podem ter câncer de mama, mas é raro, corresponde apenas $1 \%$ dos casos.

Os fatores de risco também foram apresentados aos participantes, sendo eles: a idade, pessoas idosas têm maior possibilidade da doença; comportamentais/ambientais: excesso de bebida alcoólica, sedentarismo e má alimentação; história reprodutiva/hormonais: primeira menstruação (menarca) antes de 12 anos, não ter tido filhos, primeira gravidez após os 30 anos, menopausa após os 55 anos e à reposição hormonal pós-menopausa, por mais de cinco anos; hereditários/genético: histórico familiar, como câncer de ovário, câncer de mama em homens e câncer de mamas em mulheres. Foi bem enfatizado nessa parte que por ter um ou mais desses fatores de risco a mulher não necessariamente desenvolveria o câncer.

Com base em informações do INCA foi apresentado quais os sintomas as mulheres deveriam estar atentas para um possível câncer de mama, dentre esses sintomas foi mostrado: o nódulo sendo a causa mais comum e que é percebido muitas vezes pela própria mulher, mostrando a importância das mesmas estarem atentas, secreção ou perda de líquido pelo mamilo, alteração da cor, espessura ou textura da pele da mama, retração da pele e retração ou alteração de posição ou forma do mamilo, foi destacado que qualquer aparição de algum desses sintomas devem ser investigados por um médico.

Com a apresentação dos sintomas foi feita a correlação para mostrarmos a importância das mulheres se conhecerem por meio do auto exame, enfatizando que as mulheres possuírem uma postura atenta, é essencial para uma detecção precoce de câncer de mama, que é importante que as mulheres se observem e se toquem, e notem assim, qualquer alteração em suas mamas. Neste momento, foi ensinado as mulheres a como realizar o autoexame nelas mesmas, uma integrante colocou a mama amiga (Figura 2), e mostrou passo a passo a maneira correta de como deveriam fazer, mostrando a importância de olhar, sentir e apalpar as suas mamas, esse foi um dos pontos altos da palestra pelo fato de mostrar as mulheres que eles devem sim se conhecer e "se sentir" sem vergonha ou medo. 
Figura 2 - Discente com a mama amiga

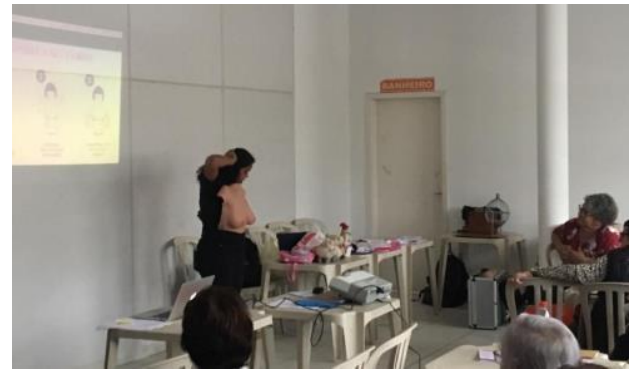

Fonte: Acervo pesquisa (2019).

A prevenção foi um dos principais tópicos destacados, visto que cerca de $30 \%$ dos casos de câncer de mama podem ser evitados com a adoção de hábitos saudáveis (INCA, 2018), como: prática de atividade física, alimentação saudável, peso corporal adequado, evitar o consuma em excesso de bebida alcoólica e amamentar o máximo de tempo possível é um fator de proteção para o câncer.

Um ponto importante foi a questão de quando procurar o médico, ou seja, em caso de identificação de alguma alteração pela própria mulher através do autoexame, a mulher deve procurar o quanto antes os serviços de saúde mais próximo para avaliação diagnóstica. Na Figura 3, é visto as etapas quando o indivíduo percebe alguma alteração na mama, geralmente este é encaminhado até o posto de saúde, onde terá um consulta com um médico clínico geral que possivelmente pedirá ou indicará uma mamografia, caso tenha alguma alteração nesta mamografia, a paciente será encaminhada para um especialista, o mastologista.

Figura 3- Linha de cuidado do câncer de mama

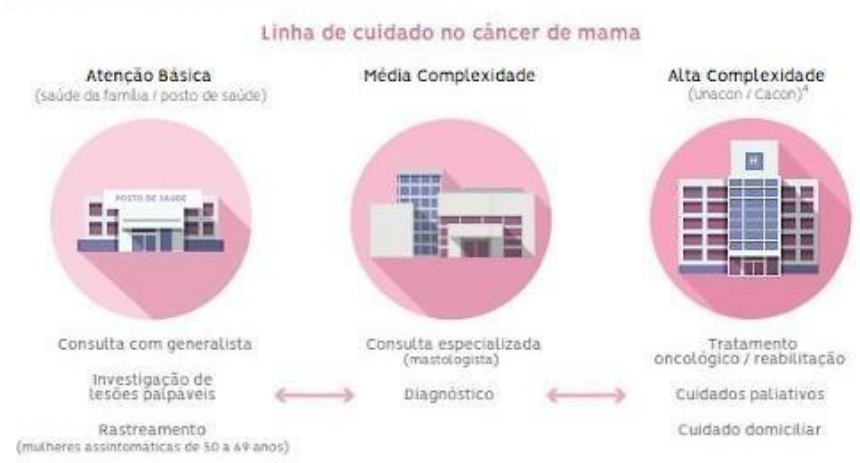

Fonte: INCA (2019).

O diagnóstico das alterações suspeitas nas mamas são feitos por meio dos seguintes exames: mamografia, é uma radiografia das mamas feita por um equipamento de raios X, chamado mamógrafo, capaz de identificar alterações suspeitas de câncer antes do surgimento dos sintomas, ou seja, antes que seja palpada qualquer alteração nas 
mamas. O exame ultrassonográfico é muito importante na detecção de alterações mamárias variadas e é feita em mulheres jovens por causa de sua densidade mamária que não é recomendado à mamografia. À confirmação do diagnóstico é feita por meio da biópsia, que consiste na retirada de um pedaço do nódulo/lesão, que será enviada para análise.

O tratamento varia conforme a fase do tumor (estadiamento), o tipo de tumor e as condições da paciente. Às modalidades de tratamento, são: tratamento local (cirurgia e radioterapia) e tratamento sistêmico (quimioterapia e hormonioterapia). Em muitos casos, é necessário combinar mais de uma modalidade. A cirurgia oncológica é um tipo de tratamento do câncer que retira o tumor através de um operação. À quimioterapia é um tratamento em que se utilizam medicamentos para combater o câncer, eles se misturam com o sangue e se espalham pelo corpo, destruindo as células doentes (tumor) e impedindo que se espalhem. A radioterapia é um tratamento que utiliza radiações ionizantes (raio x), um tipo de energia para destruir ou impedir que as células do tumor aumentem.

E para finalizar a palestra foi destacado sobre a reconstrução mamária, focando nos direitos da mulher de acordo com base na Lei $\mathrm{n}^{\circ} 12.802$, onde se estabelece que a reconstrução mamária é um direito da mulher que teve a mama retirada pelo tratamento do câncer e que ela deve ser feita imediatamente após a retirada das mamas se existirem condições técnicas para isso. No caso de impossibilidade da reconstrução imediata, a paciente tem direito a acompanhamento e realização da cirurgia assim que ela alcançar as condições requeridas para a reconstrução. Para solicitar à reconstrução pelo SUS, a paciente pode agendar a cirurgia de reconstrução mamária no local do tratamento, caso a paciente não estiver mais em tratamento, deverá se dirigir a uma Unidade Básica de Saúde e solicitar o seu encaminhamento para uma unidade especializada em cirurgia de reconstrução mamária. A paciente também tem o direito a cirurgia plástica de correção de eventual assimetria entre a mama afetada pelo câncer e a mama saudável.

\section{DINÂMICA}

Para a atividade se tornar atrativa aos idosos entre os assuntos de câncer de mama e osteoporose, foi realizado um sorteio, com números que estavam grampeados ao folder (Figura 04-c) que foi disponibilizado para cada participante, foram 4 livros e 4 canetas recicláveis (Figura 04-a, b e d) doados para o projeto. À decisão desta dinâmica entre às apresentações se deu pelo fato de se tratar de participantes idosos, que se cansam 
rapidamente, ou seja, foi um momento de descontração e descanso entre uma apresentação e à outra.

Figura 04 - Palestra oferecida na comunidade no ano de 2019: (a) e (b) Livros; (c) Folder com número para sorteio; (d) Caneta para sorteio

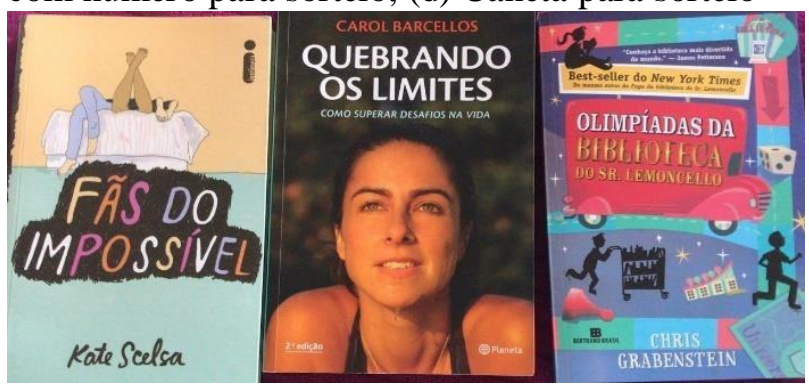

(a)

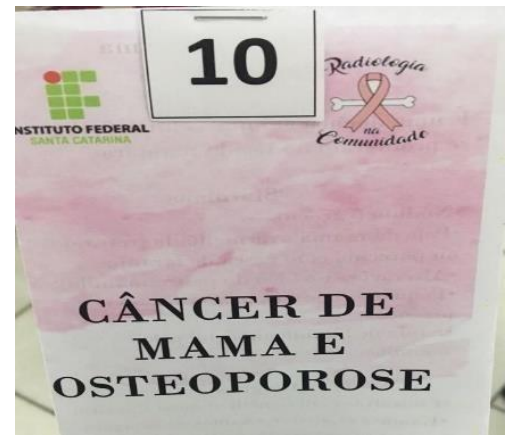

(c)

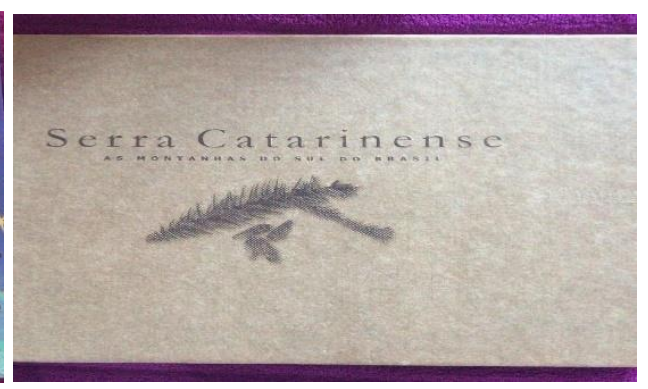

(b)

Fonte: Do Autor (2019).

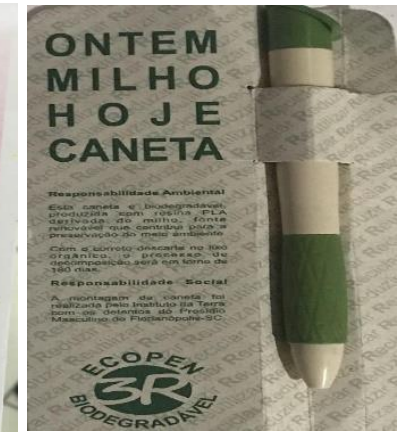

(d)

\section{OSTEOPOROSE}

A apresentação sobre a osteoporose foi iniciada com os principais elementos que compõem o tecido ósseo, sendo eles o cálcio, o colágeno e o fósforo. A parte interna do osso é denominada matriz óssea, esta representa a flexibilidade, a rigidez e a resistência dos ossos. Em seguida foi abordado que a osteoporose é uma doença óssea que provoca a diminuição de absorção de minerais e de cálcio. Para incentivar a participação dos ouvintes, foi questionado ao público por qual motivo esta doença atinge especialmente as mulheres, eles não souberam responder à esta pergunta, contudo se interessaram para saber o motivo, desta forma, foi explicado que isto ocorre, devido as mulheres terem menor massa óssea em qualquer idade quando comparado com os homens, além disto, taxa de perda de massa óssea nas mulheres é maior do que nos homens e aumenta após a menopausa. Foi apresentado um dado que de acordo com Silva (2012) durante toda sua vida as mulheres perdem cerca de $40 \%$ de sua massa óssea, enquanto que os homens perdem cerca de $25 \%$.

Foi explicado para a comunidade que a osteoporose apresenta estágios. A osteopenia é uma condição pré-clínica que sugere a perda gradual de massa óssea que pode levar à osteoporose, comprometendo a resistência dos ossos e por consequência

Cidadania em Ação; Revista de Extensão e Cultura, Florianópolis (SC), v. 3, n. 2, jul./dez. 2019 
aumentando o risco de fraturas, foi mostrado através de um esquema de imagens didáticas, como fica a aparência de um osso normal, de um osso com osteopenia e de um osso com osteoporose. A perda de massa óssea não apresenta sintomas, então quando está num estágio significativo já acarreta alterações clínicas.

Durante o questionário aplicado, percebeu- se que a maioria dos idosos conheciam e/ ou já realizaram o exame de densitometria óssea. Foi mencionado que este é o principal exame para diagnosticar a osteoporose, pois possibilita medir a densidade mineral do osso de uma ou mais regiões anatômicas do paciente, para que os valores possam ser comparados com valores de referência já pré-estabelecidos.

Foi abordado também sobre quais as considerações necessárias para realizar o exame. Foi explicado que mulheres grávidas, pessoas que tem impossibilidade de manterse na posição durante o exame; pessoas que realizaram ingestão recente de meio de contraste ou realizaram exame de medicina nuclear são contraindicadas para realização do exame. Além disto, o grupo explicou que antes de entrar na sala de exame, o paciente é entrevistado e responde uma série de perguntas, tais como: nome, idade, peso e altura,

sexo, qual motivo da realização do exame, se tem exames anteriores, se apresenta problemas de saúde, se tem prótese ou fez cirurgia, se já fraturou algum osso, se fuma ou ingere bebidas alcoólicas diariamente. Foi explicado que o paciente não deve ingerir medicamentos que contenham cálcio 24 horas antes de realizar o exame e que a troca de roupa por um avental é importante para que o exame seja o melhor possível para laudos.

Os fatores de risco também foram apresentados aos participantes, de acordo com o artigo de Souza (2010), os fatores de risco mais valorizados para osteoporose são: o gênero feminino, as etnias amarela e branca, a idade mais avançada, a precocidade do início da menopausa, a hereditariedade (presença de osteoporose ou de fratura osteoporótica entre os ancestrais e os colaterais), história pregressa de fraturas osteoporóticas, erros nutricionais (baixa ingestão de cálcio, baixa ingestão de vitamina D3 ou baixa insolação para produção da mesma, situações para má absorção de alimentos etc.), maus hábitos (ingestão exagerada de café, álcool, tabaco), sedentarismo, certas medicações (glicocorticóides, anticonvulsivantes) e doenças como a artrite reumatoide e quase todas as doenças inflamatórias sistêmicas.

Medidas de prevenção, que consistem basicamente em evitar a exposição aos fatores de risco, se alimentar de forma saudável e buscar alimentos com fontes ricas em cálcio, vitamina D, evitar o consumo excessivo de bebidas alcoólicas, tabaco, café e 
refrigerantes, bem como praticar exercícios físicos regularmente. Também, medidas de tratamento foram abordadas, tais como, suplementação de cálcio, vitamina $\mathrm{D}$, terapia de reposição hormonal e estrogênica (TRH e TRE), além da atividade física.

\section{DINÂMICA}

No dia 12 de abril de 2019, a organizadora do Grupo de Convivência Para Idosos informou aos organizadores do projeto que os participantes gostam de jogar bingo, por isso a dinâmica elaborada pelo grupo, foi toda pensada e esquematizada de acordo com as características que à comunidade em questão apresenta. Com isso os organizadores do projeto desenvolveram cartelas do bingo diferenciadas com um conjunto de 40 palavras sobre os temas (Figura 05), às palavras utilizadas nas cartelas apareceram durante as apresentações.

Figura 05- Modelo Cartela

\begin{tabular}{|c|c|c|}
\hline \multicolumn{3}{|c|}{ BINGO DA SAÚdE } \\
\hline LEI N'12.802 & PESO & MENOPAUSA \\
\hline $\begin{array}{c}\text { DIAGNÓSTICO } \\
\text { RADIOLOGIA } \\
\text { IFSC }\end{array}$ & CIRURGIA \\
\hline $\begin{array}{c}\text { DENSIDADE } \\
\text { DO OSSO }\end{array}$ & MAMA & FUMO \\
\hline
\end{tabular}

Fonte: Acervo pesquisa (2019).

Para presentear os ganhadores das rodadas do bingo os discentes usaram as doações feitas pelo comércio local, a Figura 6 mostra alguma dessas doações.

Figura 06: Doações
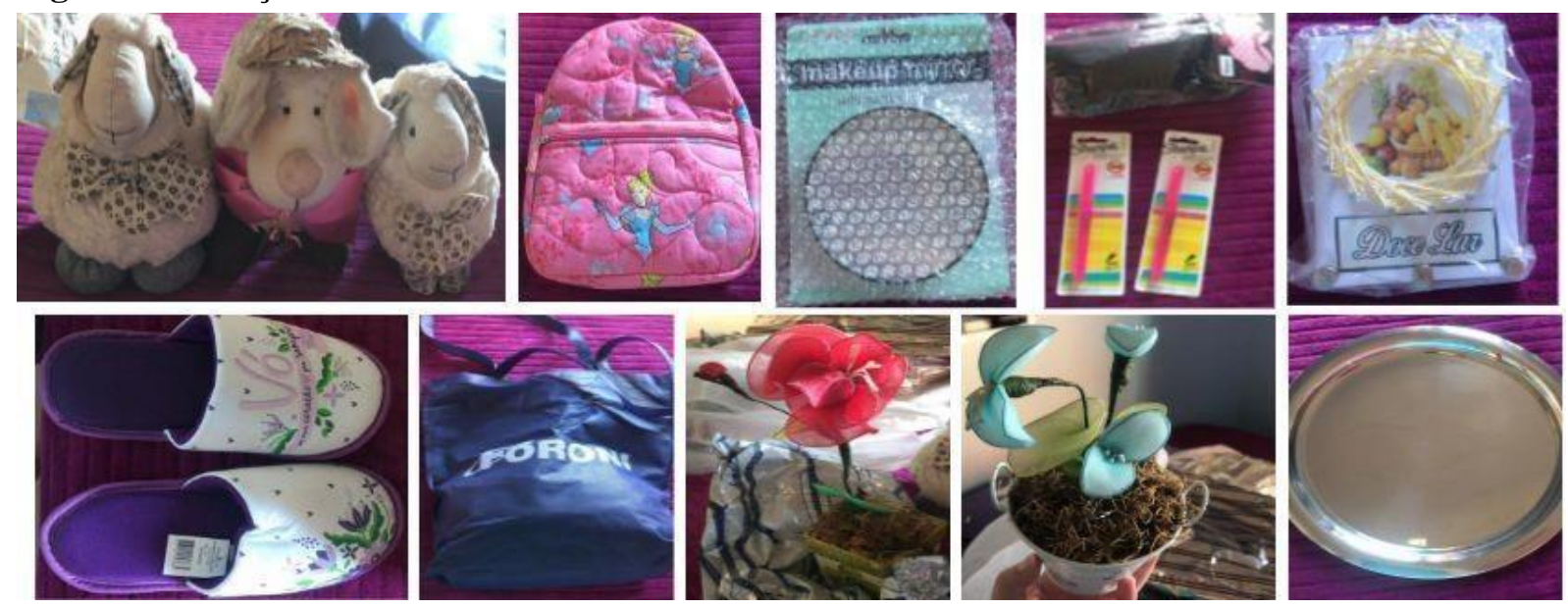

Fonte: Acervo da pesquisa (2019). 
$\mathrm{Na}$ primeira rodada do bingo os participantes teriam que marcar três palavras na primeira coluna na vertical, onde o prêmio era um conjunto de três ovelhas decorativas doados para o projeto.

$\mathrm{Na}$ segunda rodada do bingo os participantes teriam que marcar três palavras na última linha horizontalmente, onde o prêmio era uma mochila da cinderela junto com um espelho e um porta chave doados para o projeto.

$\mathrm{Na}$ terceira rodada do bingo os participantes teriam que marcar quatro palavras conforme o modelo, onde o prêmio era uma bolsa com dois marca textos, um estojo e uma bandeja de inox doados para o projeto.

$\mathrm{Na}$ quarta rodada do bingo os participantes teriam que marcar uma palavra em cada ponta da cartela, o prêmio era uma pantufa doados para o projeto.

$\mathrm{Na}$ quinta rodada do bingo os participantes não poderiam marcar nenhuma palavra na cartela, esse método é conhecido como azarão, onde quem não marca nada ganha o prêmio, o prêmio era dois vasos de flores artificiais doados para o projeto, nessa rodada ao final ficaram duas participantes que decidiram dividir o prêmio.

Após as rodadas de bingo, os organizadores fizeram mais dois sorteios com os números que estavam grampeados ao folder que foi disponibilizado para cada participante. Os prêmios foram uma camiseta da radiologia na comunidade e uma bolsa também da radiologia na comunidade.

Figura 7- Palestras oferecidas nas comunidade no ano de 2019, prêmios do sorteio
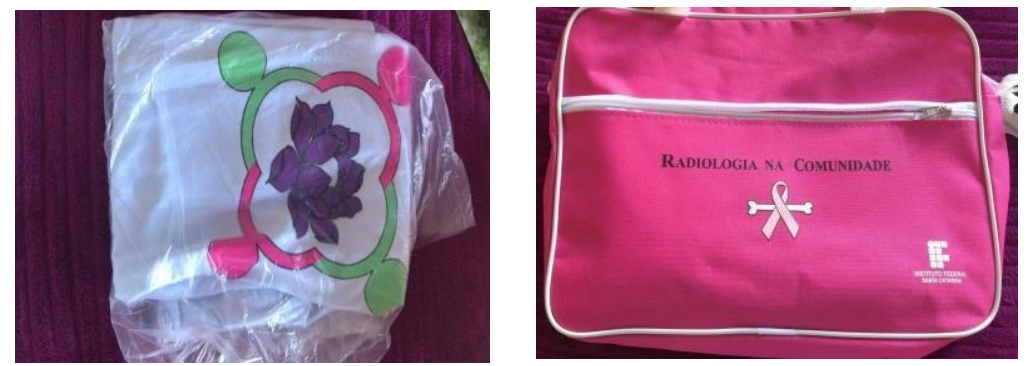

Fonte: Acervo pesquisa (2019).

Com a finalização das apresentações, do bingo e dos sorteios, a equipe do projeto entregou ao participantes uma lembrancinha com agradecimento de participação que foi confeccionada pela própria equipe. Após os agradecimentos, o Grupo de Convivência Para Idosos convidou os organizadores do projeto e as docentes orientadoras a se juntar com eles para um café coletivo, nesse café foi possível uma troca de experiência ainda mais próxima com os idosos, além de conversas informais sobre o tema que mostrou o 
quanto eles estavam atentos e interessados na palestra. Ao final da confraternização foi pedido autorização para fotos com o grupo de idosos.

Figura 8 - Palestras oferecidas nas comunidades no ano de 2019:

(a) Lembrancinha;

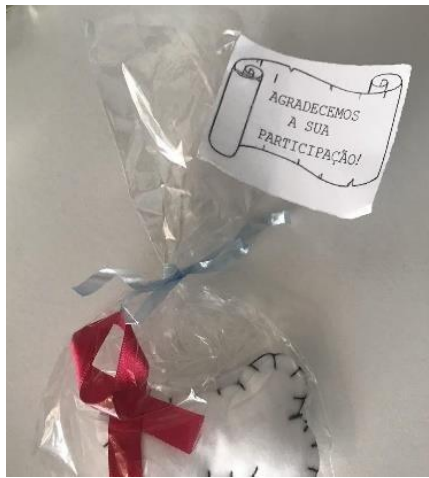

Fonte: Acervo pesquisa (2019). (b) Discentes, docentes e comunidade;

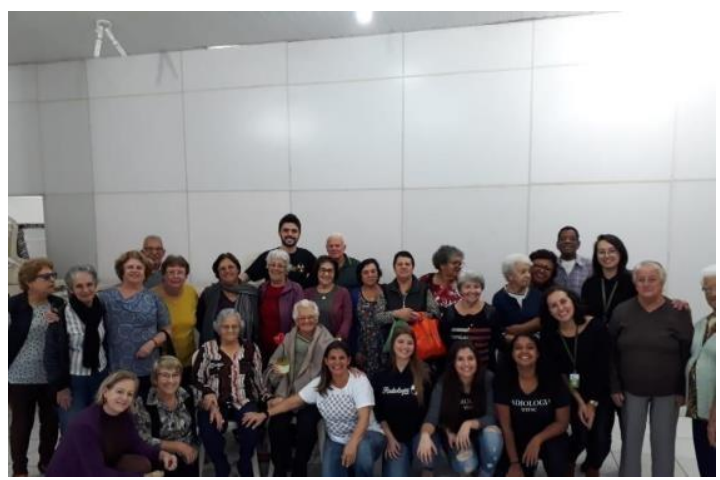

\section{RESULTADOS}

É de grande importância a conscientização das comunidades sobre a prevenção do câncer de mama e da osteoporose. Antes de dar início a apresentação, os discentes aplicaram um questionário com os participantes e a partir dele foram obtidos alguns dados, dentre eles que o público têm um bom nível de escolaridade, onde apenas um participante é analfabeto e a grande maioria tem um ensino fundamental incompleto, como demonstra o Gráfico 1.

Gráfico 1 - Grau de escolaridade dos participantes

Escolaridade

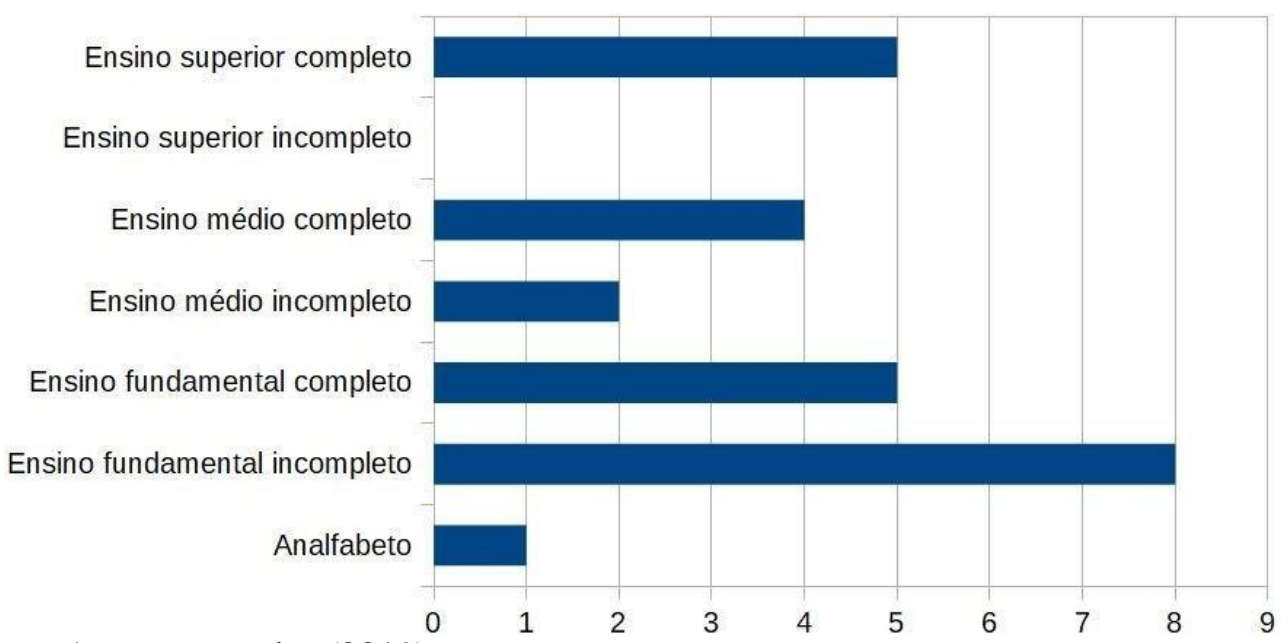

Fonte: Acervo pesquisa (2019).

No dia 24 de maio, compareceram 28 pessoas do Grupo de Convivência Para

Cidadania em Ação; Revista de Extensão e Cultura, Florianópolis (SC), v. 3, n. 2, jul./dez. 2019 
Idosos, onde 93,33\% eram do sexo feminino e 6,67\% do sexo masculino (Gráfico 2). A maioria dos participantes tinham um bom nível de escolaridade, isto foi importante, pois durante a palestra pudemos ter grandes trocas de conhecimento.

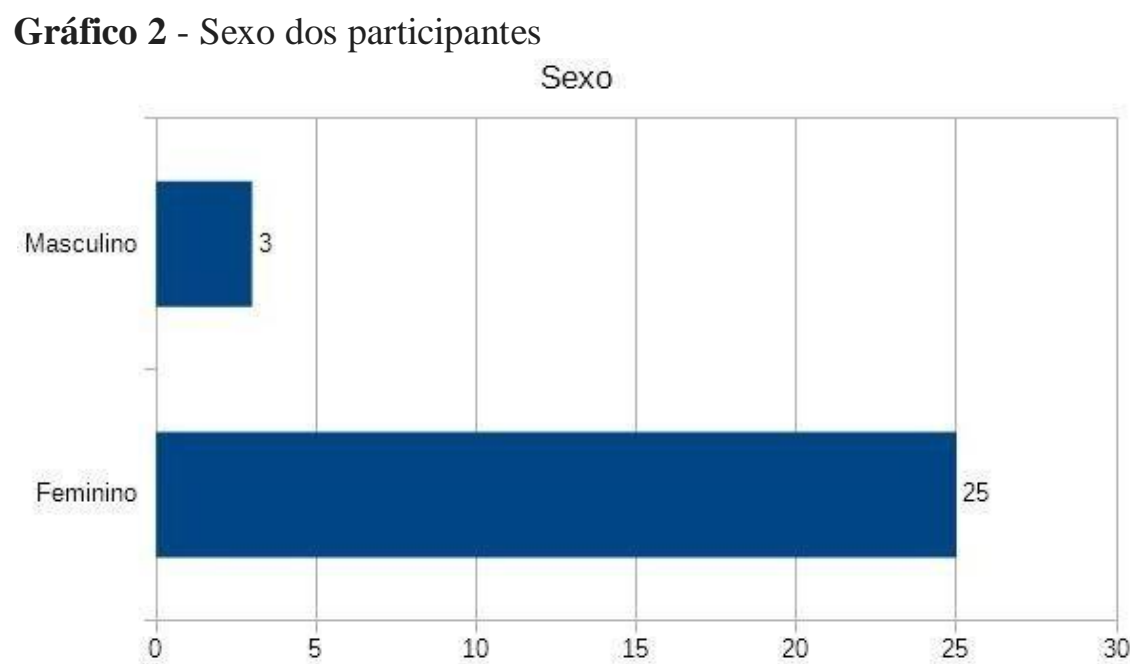

Fonte: Acervo pesquisa (2019).

O câncer de mama é um tumor maligno que se desenvolve quando há uma multiplicação desordenada das células da mama, onde estas se tornam anormais e assim formam o tumor. É importante lembrar que cada câncer evolui de uma forma diferente, uns mais lentos, outros mais rápidos. De acordo com o Instituto Nacional do Câncer, o câncer de mama é o mais comum entre as mulheres no mundo e no Brasil, diante disto, foi importante mencionar no questionário quantas pessoas já apresentaram câncer de mama em algum momento de sua vida.

De acordo com o Gráfico 3, diante o questionamento sobre o participante ter/teve câncer de mama, dos 25 participantes que responderam se já tiveram ou não câncer de mama, 03 pessoas responderam que já tiveram câncer de mama e todas estas do sexo feminino. 
Gráfico 3 - Número de participantes que apresentam ou já apresentaram câncer de mama em algum momento da vida.

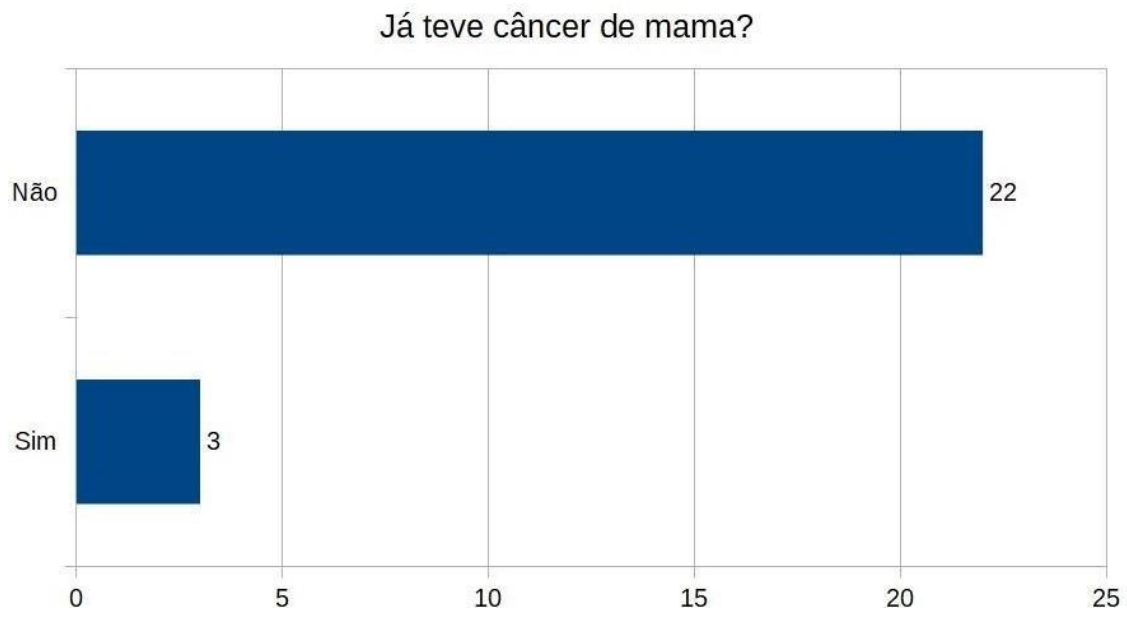

Fonte: Acervo pesquisa (2019).

Sabendo que a hereditariedade é um fator de risco à predisposição do câncer de mama, foi questionado se as pessoas já tiveram casos de câncer de mama na família. Das 25 pessoas que responderam, todas as 06 pessoas que afirmaram ter caso de câncer de mama na família, eram mulheres (Gráfico 4).

Gráfico 4 - Número de participantes em que teve familiar que apresentou caso de câncer de mama em algum momento da vida.

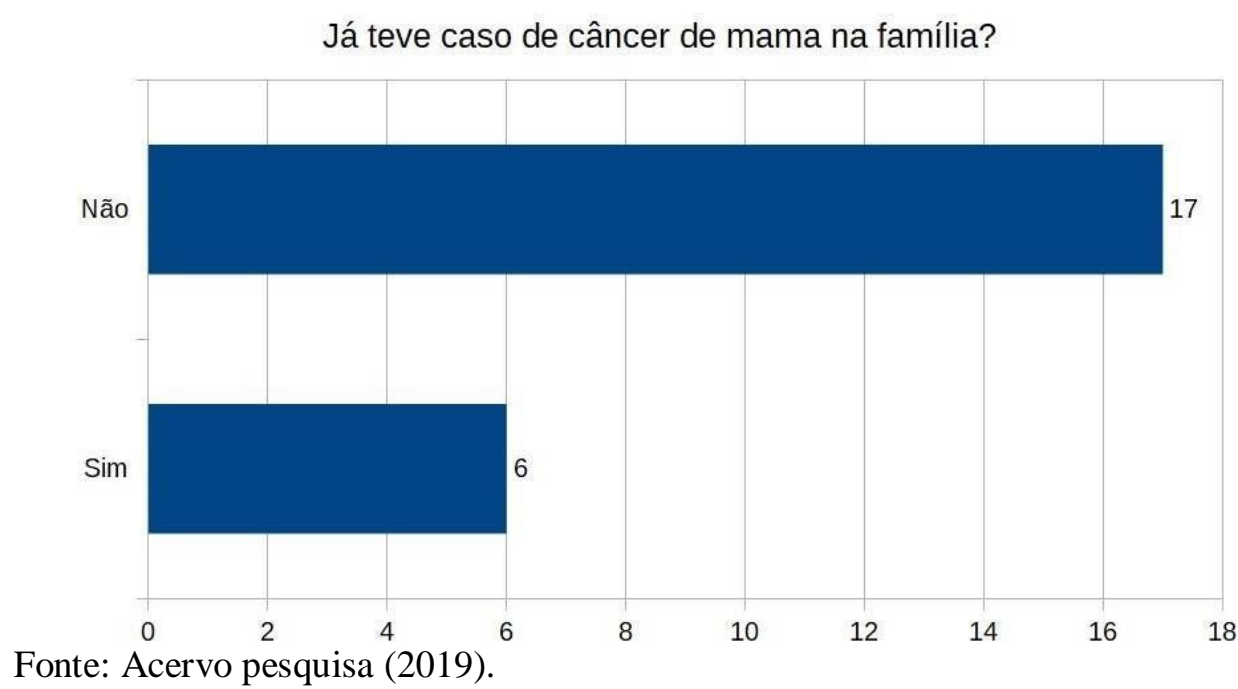

A osteoporose é uma doença crônica progressiva que é caracterizada pela diminuição da massa óssea e rompimento da arquitetura óssea, desta forma, compromete a força do osso e propicia fraturas. É relevante conhecer sobre a osteoporose pois ela é uma doença silenciosa, os sinais geralmente aparecem quando está em uma fase mais

Cidadania em Ação; Revista de Extensão e Cultura, Florianópolis (SC), v. 3, n. 2, jul./dez. 2019 
avançada. Por esse motive, questionamos quantas pessoas já tiveram esta doença, foi obtido que 13 pessoas já tiveram ou têm a osteoporose, e todas são mulheres (Gráfico 5).

Gráfico 5 - Número de participantes que apresentam ou já apresentaram osteoporose em algum momento da vida.

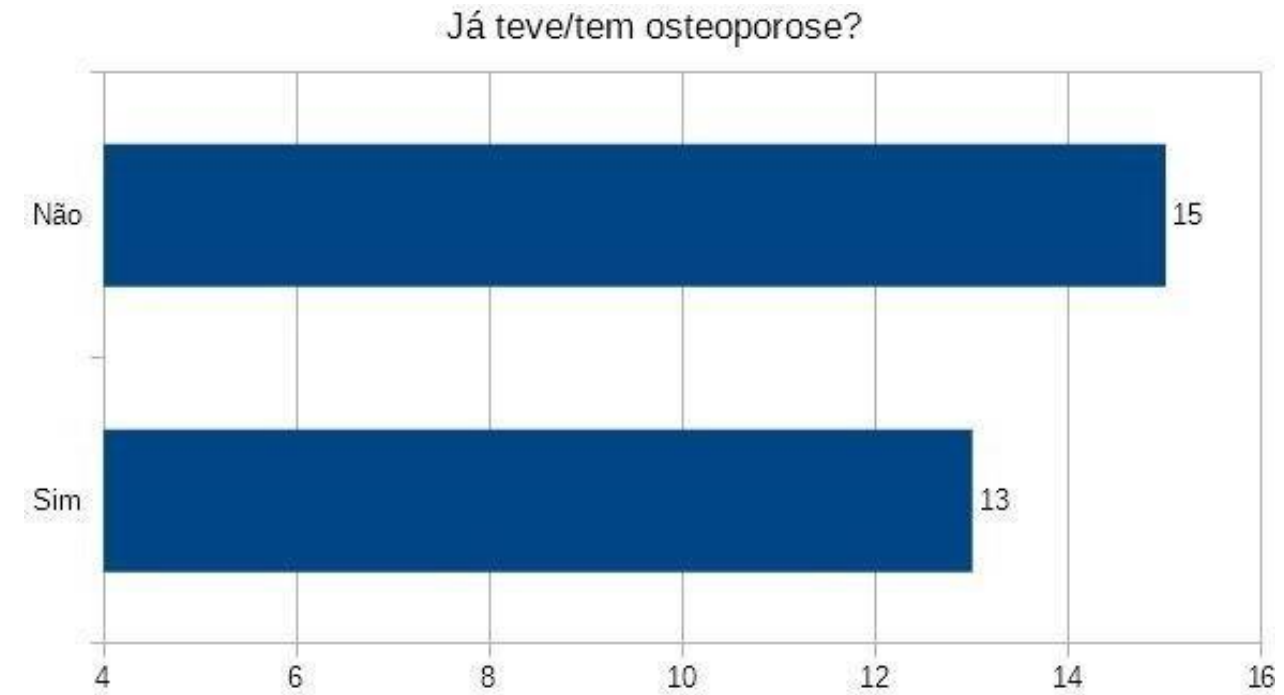

Fonte: Acervo pesquisa (2019).

Diante o questionado de se as pessoas já tiveram casos de osteoporose na família, 14 pessoas deram resposta positiva, sendo 13 do sexo feminine. Esta questão é de extrema importância sabendo que a hereditariedade é um fator de risco à predisposição da osteoporose.

Gráfico 6 - Número de participantes em que teve familiar que apresentou caso de osteoporose em algum momento da vida.

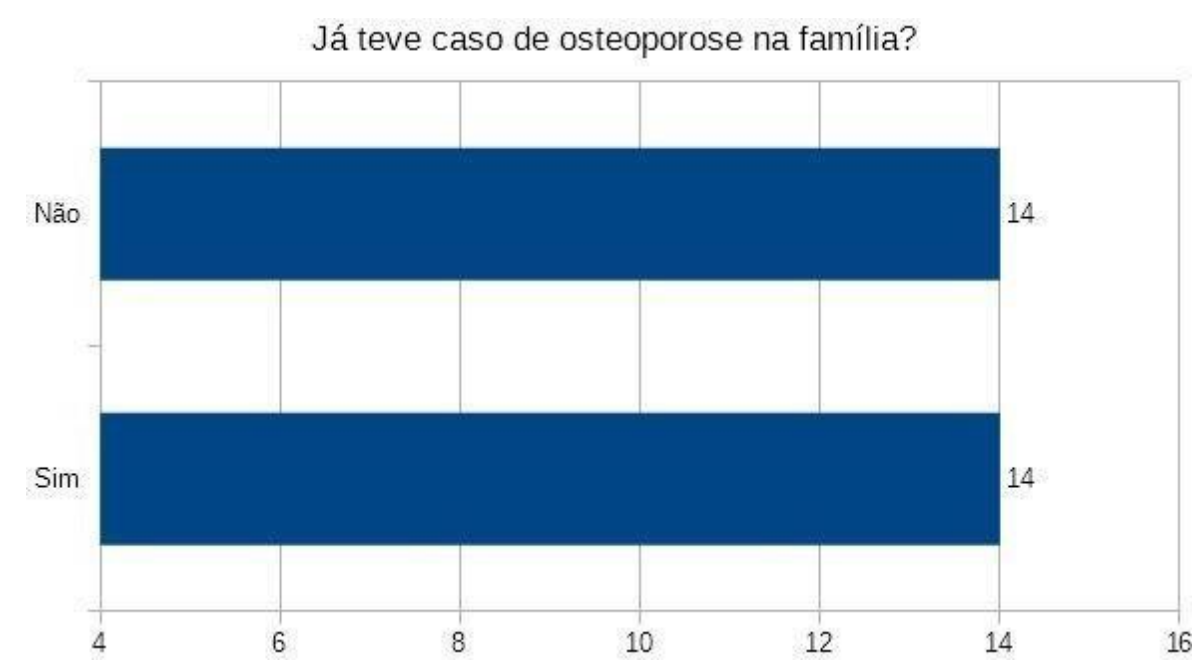

Fonte: Acervo pesquisa (2019). 
A densitometria óssea (DO) é o exame mais utilizado para o diagnóstico da osteoporose, pois ele permite avaliar a densidade mineral óssea, partindo deste ponto, foi questionado aos participantes se eles já realizaram este exame. Como resultado do questionário, das 28 pessoas que responderam, 17 afirmaram que já realizaram o exame de DO, todas mulheres (Gráfico 7).

Gráfico 7 - Número de participantes que já se submeteram ao exame de densitometria óssea.

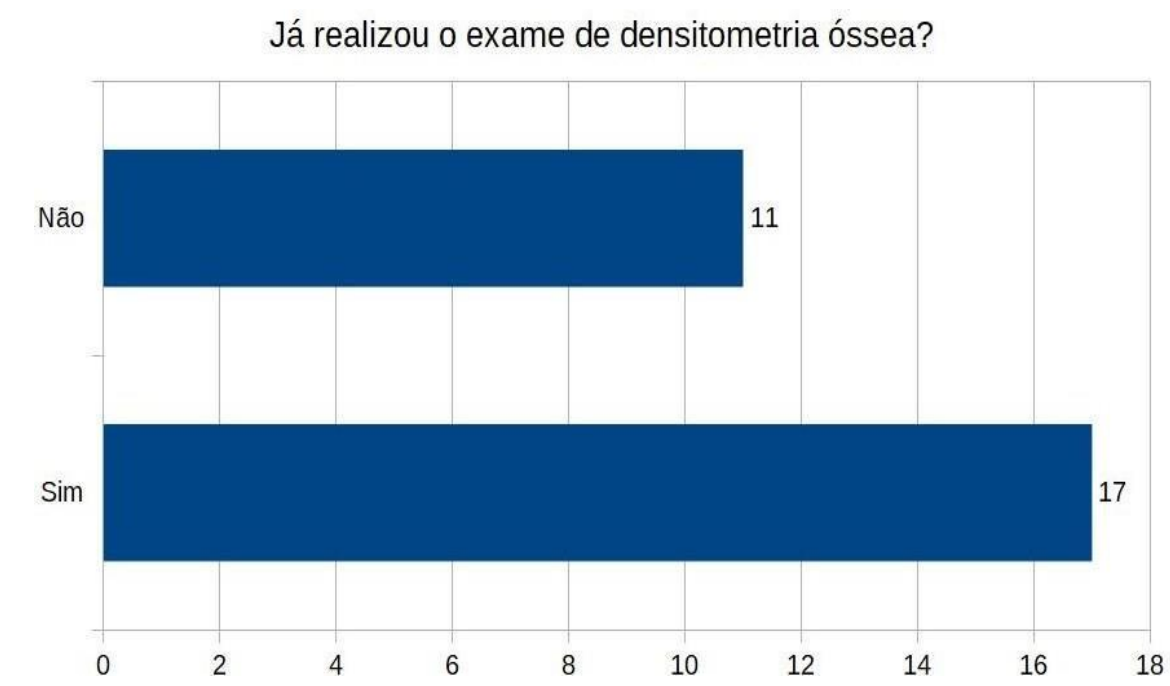

Fonte: Acervo pesquisa (2019).

\section{CONSIDERAÇÕES FINAIS}

O projeto Radiologia na Comunidade vêm atuando, desde 2006 e levando informações de qualidade sobre câncer de mama e osteoporose para diversas comunidades da região da Grande Florianópolis.

A unidade curricular do Curso Superior de Tecnologia em Radiologia denominado "Projeto de Extensão II", permitiu com que os discentes pudessem transmitir todo conhecimento necessário sobre estas duas patologias que atingem um grande número da população. Como os idosos sofrem com diversas modificações biológicas, fisiológicas, cognitivas, patológicas e socioeconômicas, logo, é de extrema importância a inclusão e uma atenção especial a esta classe, sendo o principal objetivo desta ação extensionista, transmitir conhecimento reconhecendo a importância do ciclo da vida e integralizando a comunidade.

O projeto proporcionou experiências e trocas de conhecimento entre todos os presentes na palestra, principalmente promovendo o enriquecimento profissional e acadêmico ao estudante.

Cidadania em Ação; Revista de Extensão e Cultura, Florianópolis (SC), v. 3, n. 2, jul./dez. 2019 


\section{REFERÊNCIAS}

ALMEIDA, L. E. de et al. Abordagem do tabagismo em uma sala de espera: uma experiência extensionista. Extensio: Revista Eletrônica de Extensão, [s.l.], v. 15, n. 28, p.127-136, 2018.

COELHO, J. A; MEDEIROS, C. Radiologia e comunidade: Extensão em um curso superior de tecnologia. In: Simpósio Internacional sobre interdisciplinaridade no Ensino, na Pesquisa e na Extensão-Região Sul, 2014, Florianópolis.

COSTA, A. L. D. et al. Osteoporose na atenção primária: uma oportunidade para abordar os fatores de risco. Revista Brasileira de Reumatologia, [s.1], v. 56, n. 2, p.111-116, 2016.

INCA, Instituto Nacional de Câncer. Câncer de mama: vamos falar sobre isso? 2018. Disponível em: <https://www.inca.gov.br/es/node/2350>. Acesso em: 17 jun. 2019.

INCA, Instituto Nacional de Câncer. A situação do câncer de mama no Brasil: Síntese de dados dos sistemas de informação. 2019. Disponível em:

<https://www.inca.gov.br/sites/ufu.sti.inca.local/files//media/document//a_situacao_c a_ma ma_brasil_2019.pdf>. Acesso em: 17 jun. 2019.

INCA, Instituto Nacional de Câncer. Tipos de câncer: Câncer de mama. 2019. Disponível em: <https://www.inca.gov.br/tipos-de-cancer/cancer-de-mama>. Acesso em: 17 jun. 2019.

MARTINS, J. J. et al. Educação em saúde como suporte para a qualidade de vida de grupos da terceira idade. Revista Eletrônica de Enfermagem. [s.1], v. 9, n. 2, p. 443-456, 2007. Disponível em:

http://www.fen.ufg.br/revista/v9/n2/v9n2a12.htm

MEDEIROS, C. de et al. Projeto De Extensão - Radiologia e Comunidade: Avaliação das Atividades de Promoção de Saúde na Comunidade. Extensio: Revista Eletrônica de Extensão, [s.1.], v. 11, n. 18, p.80-93, 2014.

RODRIGUES, A. L. L. et al. Contribuições da extensão universitária na sociedade.

Cadernos de Graduação - Ciências Humanas e Sociais. Aracaju, v.1, n.16, p. 141-148, 2013. Disponível em:

https://periodicos.set.edu.br/index.php/cadernohumanas/article/viewFile/494/254>. Acesso em: 08 jun. 2018.

SOUZA, M. P. G. de. Diagnóstico e tratamento da osteoporose. Revista Brasileira de Ortopedia, [s.1.], v. 45, n. 3, p.220-229, 2010.

UFES, Universidade Federal do Espírito Santo. Pró-Reitoria de Extensão: O que é a extensão universitária. 2019. Disponível em: <http://www.proex.ufes.br/ o-que-\%C3\%A9-extens\%C3\%A3o-universit\%C3\%A1ria>. Acesso em: 17 jun. 2019. 